\title{
Design of Multiple Output DC Regulator
}

\author{
Caiyun Gui ${ }^{1}$ and Baobao Kang ${ }^{2}$
}

1.Yulin University, Yulin 719000 2, Bureau of Geographical Prospecting INC., Zhuozhou, 072751

\author{
Keywords: DC voltage regulator source; Rectifier filter; Output circuit; Simulation
}

\begin{abstract}
Multi-output DC voltage source is widely used in the daily life and teaching and scientific experiments. Multi-output DC voltage regulator has adjustable output range, stable output voltage, good controllability, high accuracy and other significant advantages, which makes the multi-output DC power supply become a hot spot of teaching experiment, scientific research and development. In order to design a practical, reliable, portable, high-precision multi-output DC voltage source, the electric supply's voltage is used as input through the power transformer, rectifier and filter circuits, and then the voltage conversion chip regulator's output is $\pm 5 \mathrm{~V}, \pm 12 \mathrm{~V}$ and adjustable output voltage is $-12 \sim+12 \mathrm{~V}$ and the voltage can work in multiple ways, and the maximum output current of each way is $1.5 \mathrm{~A}$. The design structure is simple with clear level and high cost efficiency, so it can meet the output requirements for the same DC power supply.
\end{abstract}

\section{Introduction}

With the continuous development of science and technology, the requirements for the testing of the equipment are getting higher and higher, which requires the testing equipment to provide accurate testing with high precision. In experimental and practical applications, it is necessary to achieve accurate high-precision testing. The voltage signal of the testing equipment needs to be supplied with high-precision voltage values through the circuit. However, the conventional DC voltage stabilizing so0urce has few functions and is difficult to control. The accuracy is too low and the interference is large with low reliability and much complicacy. Although with the advent of the switching power supply, it is still difficult to stabilize the large output voltage of the switching power supply, and the direct stable current and voltage of some external equipment and high-altitude placards can be more durable. Meanwhile, the frequency of the switching power supply can usually reach tens to hundreds of kilohertz, and the working frequency signal source may cause very strong interference, so it is not applicable to the amplifier device of some home appliances. In addition, the switching power supply contains a lot of electronic components, so it is more prone to cause failure, which makes the repair become cumbersome and labor-consuming. The discrete analog components are used in the design to achieve a stable output of $+5 \mathrm{~V},-5 \mathrm{~V},+12$ $\mathrm{V},-12 \mathrm{~V}$ and adjustable output $-12 \sim+12 \mathrm{~V}$ power supply through the power transformer, rectifier circuit, filter circuit and the regulator circuit. All the necessary test equipment provides the stable DC voltage signal and reliable power supply with high precision, which meets the needs of voltage control. The design is simple with clear thinking and a high cost efficiency.

\section{Overall Design Scheme and Hardware Circuit Design}

Overall Structural Design. The multi-output DC voltage source system in this design mainly consists of four parts, including the step-down module, rectifier module, filter module and voltage regulator module. First of all, in order to obtain the appropriate voltage required by the circuit, it is necessary to convert the mains voltage of China into the voltage amplitude required by the circuit through the power transformer. In order to obtain the DC voltage, it is necessary to connect the bridge rectifier circuit to convert the input AC power into pulsating DC power. The rectifier circuit module of this design is the KBU610 silicon bridge rectifier, and then the filter circuit is connected to make the output voltage tend to be smooth. Although the output voltage passing the filter circuit is relatively smooth, the output voltage will still very with the fluctuations of the load. Therefore, at last, a stable circuit is needed to achieve a stable output of $+5 \mathrm{~V},-5 \mathrm{~V},+12 \mathrm{~V},-12 \mathrm{~V}$, and adjustable 
output of $-12 \mathrm{~V} \sim+12 \mathrm{~V}$ voltage power supply. System block diagram is shown in Figure 1.

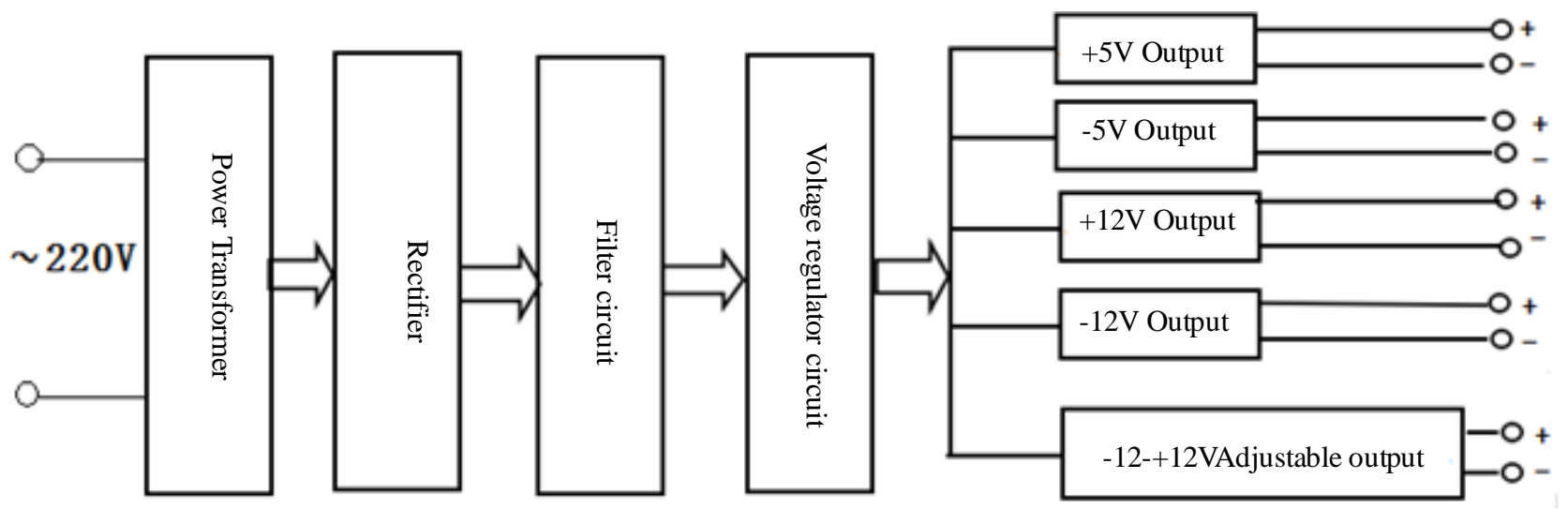

Figure 1. System Structure Diagram

Design of Rectifier Filter Circuit. The AC voltage is rectified by the rectification circuit and then output as a pulsating DC current, in which both the DC component and the AC component are present. In order to output a smoothed voltage, the waveform must be added to the filter circuit. The principle of the filter circuit is to filter out the AC component of the output voltage in the rectifier circuit by using the characteristic that the voltage across the capacitor cannot be abruptly changed and the current flowing through the inductor cannot be abruptly changed so as to keep the DC component thereof and finally obtain the smoothed voltage waveform. The design of the filter circuit design is the LC- $\pi$-type filter circuit. The advantage of this filter circuit is the combined use of inductive and capacitive filtering. This circuit performs very good filtering in passive filter circuits. Because the inductance of the AC impedance is too large, the DC resistance is small, so there is basically no loss after the DC component passes the inductor, but for the AC component, after the partial pressure jwL, most of the current drops in the inductor so that the voltage's pulse components decreases. The filter circuit has very good filter characteristics in both small and large load current, so it has strong adaptability to load and is suitable for applications with large current changes. Specific circuit is shown in Figure 2.

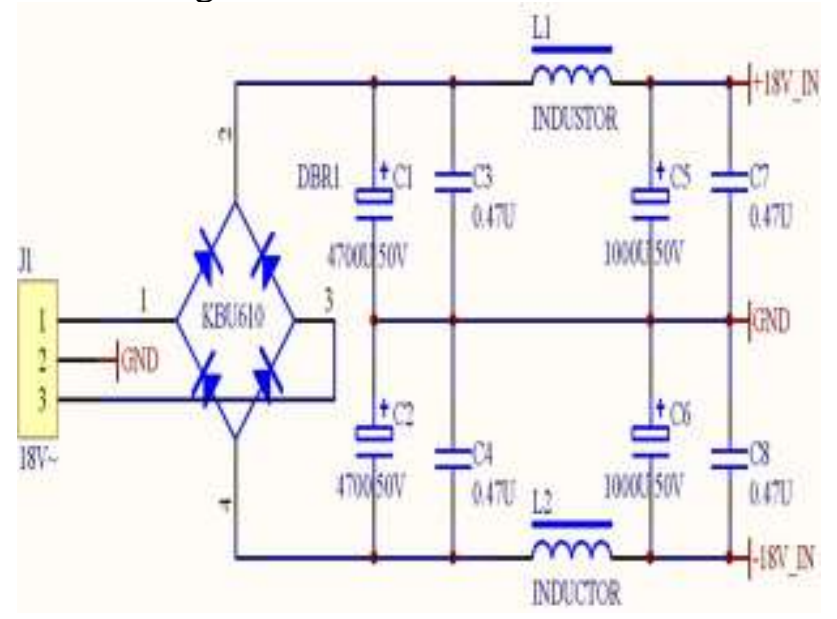

Figure 2. Rectifier Filter Circuit

Design of Voltage Output Circuit. In the voltage stabilization module, the three-terminal regulator IC LM7805 and LM7905 are used in the regulator output circuit to the output of achieve \pm $5 \mathrm{~V}$ voltage. LM7812 and LM7912 achieve the voltage of $\pm 12 \mathrm{~V}$ voltage. The voltage stabilization source composed of LM78 / LM79 series of three-terminal voltage regulator components requires very few external components, and the internal circuit is not only over-current, over-temperature protective and regulating tube protective circuit, so it both reliable and easy to use, and the price is cheap. LM317 and LM337 adjustable three-terminal regulator output range adjustable voltage's 
adjustable range is $-12 \mathrm{~V} \sim+12 \mathrm{~V}$. In order to ensure the stability and high accuracy of the output voltage, the resistor must be close enough to the regulator, which is to prevent the output current error from happening and voltage should have high-precision resistance. Because the output positive and negative voltage design circuit are the same, only positive voltage design circuit and simulation design are given. Figure 3 and Figure 4 are respectively $+5 \mathrm{~V}$ output circuit and simulation results, $+12 \mathrm{~V}$ output circuit and simulation results. Figure 5 is the adjustable voltage of the LM317 and LM337 adjustable three-terminal regulator output. In order to reduce the ripple voltage on the potentiometer, a $0.47 \mathrm{uF}$ capacitor needs to be connected in parallel with the potentiometer, and a filter capacitor $\mathrm{C} 8$ in parallel is outputted.

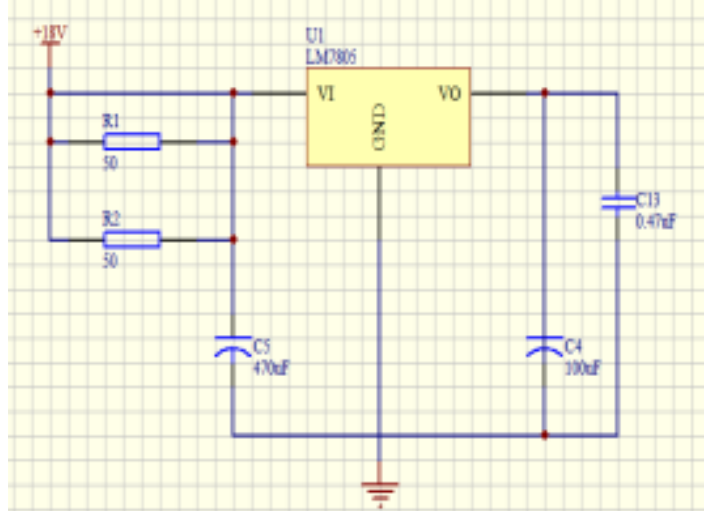

(a)+5V Output Circuit

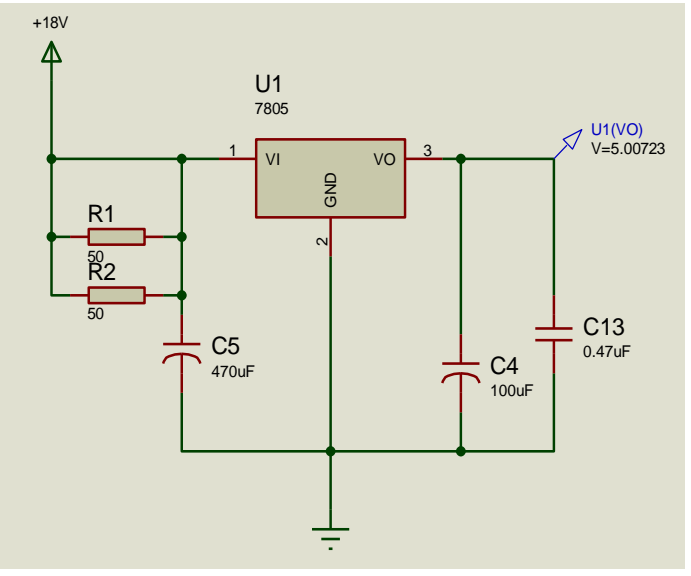

(b)+5V Simulation Results Diagram

Figure 3. +5V Output Circuit and Simulation Results Diagram

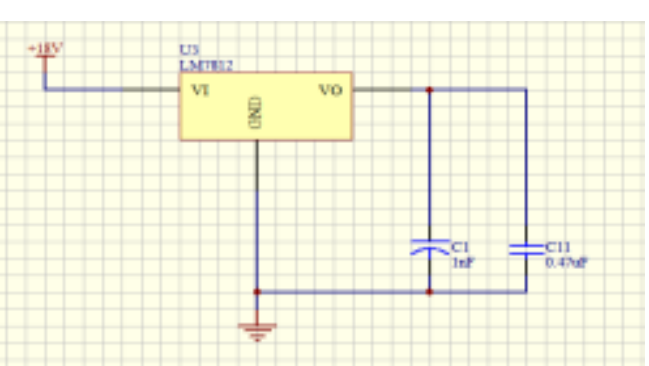

(a)+12V Output Circuit

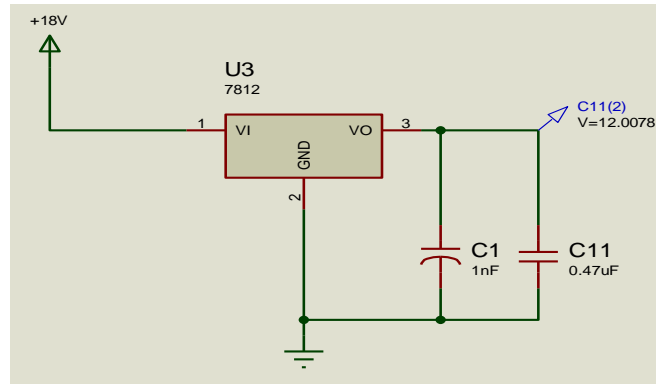

(b)+12V Simulation Results Diagram

Figure 4. $+12 \mathrm{~V}$ Output Circuit and Simulation Results Diagram
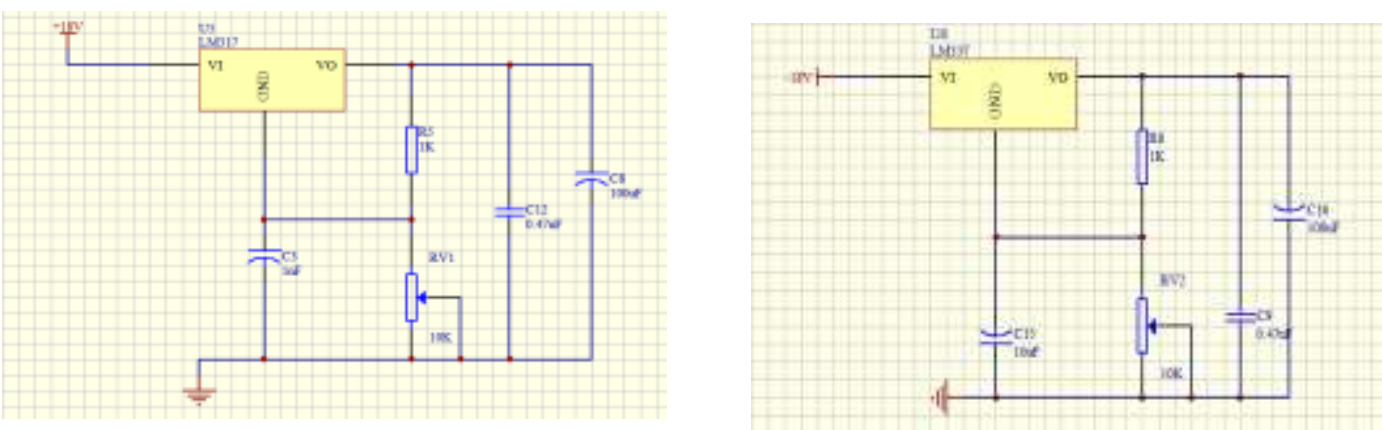

Figure 5. \pm 12 V Adjustable Output Circuit Diagram

\section{Design of Protective Circuit}

Series feedback regulator circuit has a big weakness: when the load reduces or there is a short circuit during usage, there will be a large current flowing through the adjustment tube, 
which makes it easy for the adjustment tube to be burned out, so the protective circuit must be added. There are various forms of the current protective circuit, and according to the working principles, it can be divided into current-limiting protective circuit and the ending protective circuit. When the load current exceeds the specified value, the output voltage of the power supply will drop, which ensures that the load current will not continue to increase. This is the current limiting protective circuit, as shown in Figure 6. And when the load current exceeds the maximum specified value, the voltage regulator circuit will be automatically cut off, and then the output voltage is zero or very small at this time. This is the cut-off type protective circuit, as shown in Figure 7.

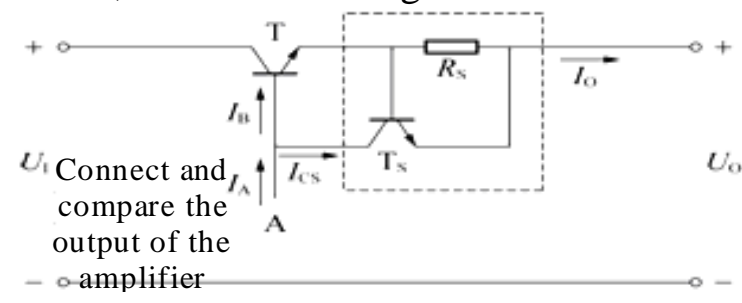

Figure 6. Current-Limiting Protective Circuit

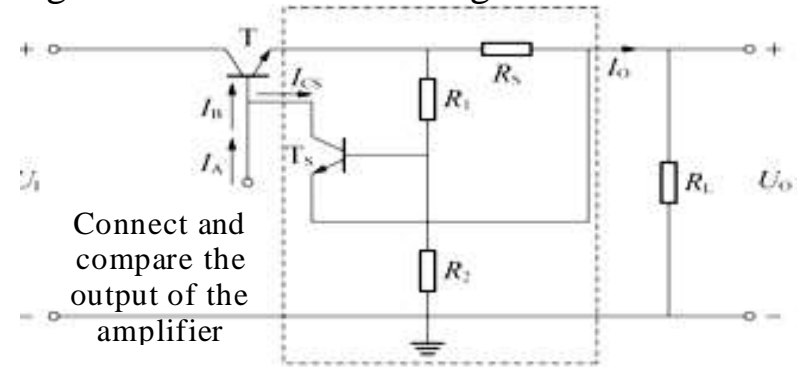

Figure 7. Cut-off Protective Circuit

\section{Conclusion}

The discrete analog components are used in this design to achieve a stable output of $+5 \mathrm{~V}$, $-5 \mathrm{~V},+12 \mathrm{~V},-12 \mathrm{~V}$ and adjustable output $-12 \sim+12 \mathrm{~V}$ power supply through the power transformer, rectifier circuit, filter circuit and the regulator circuit. The required test equipment provides stable DC voltage signal and reliable power supply with high precision to meet the required voltage control. The design is of simple structure and clear thinking with a high cost efficiency.

\section{References}

[1] $\mathrm{Hu} \mathrm{Yu,} \mathrm{Chen} \mathrm{Tao.} \mathrm{Digital} \mathrm{Filtering} \mathrm{Method} \mathrm{to} \mathrm{Restrain} \mathrm{Power} \mathrm{Frequency} \mathrm{Interference} \mathrm{in} \mathrm{ECG}$ [J]. Electronic Measurement Technology, 2011,34(8):22-26.

[2] Wu Hengyu, Tang Mintang. Design of NC DC Stabilizer Based on 89S51 Single Chip Microcomputer [J]. Manufacturing Automation, 2010,32(1):95-96.

[3] Yang Ning. Research and Realization of LXI Programmable Power Supply Based on ARM9 [D]. Xi'an: Xi'an University of Electronic Science and Technology, 2011:9-15.

[4] Cheng Xiaojie. CMOS Overcurrent Protective Circuit Design Based on Low Dropout Regulator [D]. Chengdu: Southwest Jiaotong University, 2006:12-24.

[5] Wang Zengfu, Li Chang, Wei Yongming. New Linear DC Power Supply [M]. Beijing: Electronic Industry Press, 2005. 